\title{
Effect of Vitamin C on Some Biochemical Profile in Water Deprived Goats
}

\author{
S. Kour, J. Devi ", K. Sarma and D. Chakraborty \\ Division of Veterinary Physiology \& Biochemistry, F.V.Sc. and A.H., SKUAST-J, R.S. Pura, \\ Jammu-181102, J\&K, India \\ *Corresponding author
}

\section{A B S T R A C T}

\section{Keywords}

Bio-chemical parameters, Goats, Water-deprivation, Vitamin C supplementation

Article Info

Accepted: 07 September 2020 Available Online: 10 October 2020
The effect of vitamin $\mathrm{C}$ on biochemical parameters in water deprived goats was studied. Total 18 numbers of adult male goats were selected for the experiment during summer and winter seasons. In control group ( $\mathrm{n}=6$, for each season), water was offered ad lib throughout the experimental period. Two treatment groups (T1 and T2) consisting of 6 animals each were selected. In T1 group, water was restricted and subdivided into 3 experimental periods: in period 1 (day 1 to 7 ), animals were adapted to water restriction regime by limiting access to water gradually from 15 to $3 \mathrm{hrs} /$ day. In period 2 (day 8 to 14), animals of the treatment groups had access to water for $3 \mathrm{hrs} / \mathrm{day}$. In period 3 (day 15 to 22), animals had access to water only every second day for 6 hours. In T2 group, the same protocol as mentioned above was followed with addition of vitamin $\mathrm{C}$ supplementation at a dose rate of $180 \mathrm{mg} / \mathrm{kg}$ b. wt./animal/day. Blood samples were collected on 1, 7, 14and 22 days of experiments during both seasons for the estimation of different biochemical parameters. Glucose concentrations in water deprived goats (T1 group) showed decreasing trend from day 1 to day 22 of experiment during both seasons and the values varied significantly $(\mathrm{P}<0.05)$ with each other. Significantly lower $(\mathrm{P}<0.05)$ glucose concentration was observed in water-deprived goats as compared to other two groups. Water deprivation was associated with significant $(\mathrm{P}<0.05)$ increase in serum total protein and albumin concentrations. Total serum protein and albumin concentrations were higher in water deprived (T1) goats compared to T2 and control group. In all the groups, overall summer values of total protein and albumin were found higher than winter season. The cholesterol level showed increasing trend from day 1 gradually up to day 22 in water deprived goats and all the values varied significantly $(\mathrm{P}<0.05)$ with each other in both seasons. Vitamin $\mathrm{C}$ supplemented group, cholesterol level was decreased gradually from day 7 up to day 22 during both seasons. The serum urea and creatinine levels were showed an increasing trend in water deprived goats during both seasons. The urea level showed increasing trend from day 1 gradually to day 14 , then decreased on day 22 in vitamin supplemented group during summer season. Significantly higher $(\mathrm{P}<0.05)$ urea concentration was recorded in summer than winter season in control, T1 and T2 groups. Based on the study, it can be concluded that water deprivation had negative impact on biochemical parameters, which can be ameliorated by supplementation of ascorbic acid at the rate of @ $180 \mathrm{mg} / \mathrm{kg}$ b.wt./day in goat during heat and cold stress conditions. 


\section{Introduction}

Livestock undergo various kinds of stress such as physical, nutritional, chemical, psychological and thermal. Among all, thermal stress is the most concerning now a days in the ever changing climatic scenario. Thermal stress redistributes the body resources including protein and energy at the cost of decreased growth, reproduction, production and health. Meteorological data during last 25 years indicated that the environmental temperature of the Jammu region is ranged between $4^{\circ} \mathrm{C}$ in winter to $48^{\circ} \mathrm{C}$ at summer season with relative humidity ranging from 18 to $91 \%$. Wide ranges of environmental temperature may alter the physiological status of the animal and may lead to stress condition.

Climate change is expected to account for about 20 percent of the global increase in water scarcity this century. The Intergovernmental Panel on Climate Change (IPCC) predicts that global warming will alter precipitation patterns around the world, melt mountain glaciers and worsen the extremes of droughts and floods. Countries such as India are likely to be hit hard by global warming, which will lead to serious water shortages and will affect the overall animal production. Globally livestock contribute $40 \%$ of the agricultural GDP. As the earth's temperature continues to rise, and sometimes resulting into droughts, we can expect a significant impact on our livestock sectors. The areas of $\mathrm{J} \& \mathrm{~K}$ are characterized by water scarcity and fluctuating precipitation, under the effect of global warming and unpredictable weather, rainfall is becoming even more irregular and water availability become more limited. Annual rainfall in Jammu province in the year 2015 was $1909.7 \mathrm{mms}$ with an average of $914.4 \mathrm{~mm} /$ year. Along the water accessibility, feed and other resources will be markedly affected by climate change. Keeping view on that, strategies have to be developed to use water efficiently and conservation for a diversified production system in different locations. Blood metabolites are considered to be the most accurate measure for assessing the physiological status of goats compared to other measures used such as body condition scoring and body weight gain. They are a direct measure that considers blood parameters giving the utmost possible accuracy (Madziga et al., 2013) which include glucose, blood urea, creatinine and cholesterol concentrations (Grunwaldt et al., 2005; Ndlovu et al., 2007).

Animal producer and researchers have looked for ways to alleviate the negative effects of common stressors. Stress alleviation strategies are numerous, and their availability to producers depend on the access to water and energy, the price they are able to pay and the adopted farming system. These strategies vary from simple on farm practices such as modifying the feeding pattern such as feeding of vitamin C, electrolyte therapy, etc. Very few studies have been undertaken to see the effect of vitamin $\mathrm{C}$ in water stressed pigs, Japanese quails, rabbits and broilers. Vitamin $\mathrm{C}$ is known for the function as an antioxidant mainly it act as a free radical scavenger in numerous cellular oxidation processes. Daily dose of vitamin $\mathrm{C}$ may be more helpful in increasing fat mobilization under water stress than single high doses. The results obtained on the effect of vitamin $\mathrm{C}$ on blood parameters are not very clear and need more elaborated work.

The economy of $\mathrm{J} \& \mathrm{~K}$ is still agriculture dependent and is the main occupation of majority of the rural people who earn their livelihood from it. Estimated goat population of the state as per latest available integrated sample survey is 18.136 lakh. Some biochemical studies have been conducted in different seasons in this region in Beetal and 
Toggenberg goats (Kour et al., 2015; 2017).There is paucity of information on the effect of water deprivation stress on the biochemical profile of goats with supplementation of vitamin $\mathrm{C}$ and the alleviating effects. So the present study is aimed at examining in detail the changes in some important biochemical parameters in water restricted goats during extreme climates and the likely beneficial effects, if any, produced by vitamin $\mathrm{C}$ supplementation.

\section{Materials and Methods}

The study was conducted in the Division of Veterinary Physiology \& Biochemistry, Faculty of Veterinary Sciences and Animal Husbandry, Sher-e-Kashmir University of Agricultural Sciences and TechnologyJammu, R. S. Pura, Jammu, J\&K, India.

\section{Experimental animals and grouping}

The study was conducted on 18 adult male goats of 2-3 years of age. Animals were kept in the Divisional Farm of Animal Nutrition, F.V.Sc. \& A.H., SKUAST-J, R. S. Pura (Jammu). All animals were provided with standard concentrate ration and maintained under standard managemental conditions during the experimental period. Total eighteen numbers of adult male goats were selected for the experiment. The experiment was conducted during both summer and winter seasons. In the control group $(\mathrm{n}=6$, for each season), water was offered ad libitum (24 hours) throughout the experimental period. In each season, there was two (2) treatment groups (T1 and T2) consisting of 6 animals in each group. In first treatment group (T1), water was restricted and subdivided into three experimental periods: in period 1 (experimental days 1 to 7), animals were adapted to the water restriction regime by limiting access to water gradually from 15 to 3 hour per day. During the second period of the experiment (experimental days 8 to 14 ), animals of the treatment groups had access to water for 3 hours/day. In the final period of the experiment (experimental days 15 to 22), animals had access to water only every second day for 6 hours. In second treatment group (T2), the same protocol as mentioned above was followed with addition of vitamin C supplementation at a dose rate of 180 $\mathrm{mg} / \mathrm{kg}$ body weight/animal/day.

\section{Blood collection and processing of samples}

$6 \mathrm{ml}$ of blood was collected by venipuncture aseptically from jugular vein of the goats from each group on 1, 7, 14and 22 day of experiment. For blood glucose studies, $1 \mathrm{ml}$ of blood was taken in sodium fluoride vial.Five $\mathrm{ml}$ of blood sample was allowed to clot and serum was separated and collected in storage vials and was immediately stored at $-20^{\circ} \mathrm{C}$ for further biochemical analysis.

\section{Biochemical parameters}

Blood glucose, total protein, albumin, total cholesterol, BUN (Blood Urea Nitrogen) and creatinine were analyzed as per GOD-POD end point colorimetric method (Trinder, 1969), Biuret method (Gomall, 1949 and Doumasa et al., 1971), bromocresol green method (Doumasa et al., 1971), CHOD-PAP method (Burstein et al., 1970; Roeschlau et al., 1974), GLDH- Urease method (Tiffany et $a l ., 1972$ ) and Jaffe's method (Toora and Rajagopal, 2001), respectively using analytical kits manufactured by Erba Mannheim, Solan (H.P), India. For all the observed data in the present experiment, the standard statistical procedures recommended by Snedecor and Cochran (2004) have been followed. The data were presented by showing mean and standard error. The significant differences of values for different parameters studied were assessed by the test of one way and two-way analysis of variance 
depending upon the data. The significant values of different groups and weeks were calculated by Tukey's test. All the above calculations were carried out using SPSS software version 16.0 .

\section{Results and Discussion}

\section{Blood glucose}

Blood glucose level in water deprived goats (T1 group) showed decreasing trend from day 1 to day 22 of experiment during summer and winter seasons and all the values varied significantly $(\mathrm{P}<0.05)$ with each other (Table 1). Hypoglycemia was most likely a result of the decrease in feed intake which was also observed in the same study in order to reduce the endogenous metabolic heat production and thereby reduce water required for the evaporative cooling.

Samara et al., (2012) found a significant $(\mathrm{P}<0.05)$ hyperglysemia up to $93 \%$ was in goats which could be attributed to the glucogenic effect of corticosteroids that may have been released due to the stress induced in their study; while they found a significant hypoglysemia by $20 \%$ in camel. However, Abdelatif et al., (2010) reported that the water deprivation and rehydration had no significant effect on plasma glucose level.

Blood glucose level in T2 (water deprived vitamin C supplemented) group during summer season was also showing decreasing trend from day 1 to day $14(67.75 \pm 0.77$ to $62.23 \pm 0.69 \mathrm{mg} / \mathrm{dl}$ ) and then increased to $63.27 \pm 0.69 \mathrm{mg} / \mathrm{dl}$ on 22th day (Table 1). During winter season, blood glucose level showed decreasing trend from starting to the end of experiment. Significantly lower $(\mathrm{P}<0.05)$ glucose concentration was observed in water-deprived goats as compared to other two groups.
Overall summer glucose level was found significantly lower $(\mathrm{P}<0.05)$ than winter value in control and T2 group (Table 1); whereas, in T1 group (water deprived), summer value was found lower, but the values were not varied significantly. During heat stress feed consumption decreases (also reflected in our study), which comparatively lowers the blood glucose level (Kataria et al., 2002) or it might be due to increased glucose oxidation (Collier et al., 2008) during summer stress. Decreased gluconeogenesis and glycogenolysis were observed in cows during heat stress (Itoh et al., 1998). These findings were similar to the observations of Kataria et al., (1993) in Marwari goats and Rasooli et al., (2004) in heifers and Kour et al., (2017) in Beetal and Toggenberg goats. Other reporters also revealed that the decreased level of glucose (Sejian et al., 2010; Ramana et al., 2013; Suhair and Abdalla, 2013; Indu et al., 2014; Banerjee et al., 2015) during summer season might be due to decreased in feed intake. On the other hand, Sejian and Srivastava (2009) reported that glucose level increased significantly $(\mathrm{P}<0.05)$ after thermal exposure.

\section{Serum total protein and serum albumin}

The water deprivation was associated with significant $(\mathrm{P}<0.05)$ increase in serum concentrations of total protein and albumin (Table 1). Other studies also reported that the serum protein and albumin concentrations were increased (Alamer, 2006; Casamassina et al., 2008; Ghanem et al., 2008; Hamadeh et al., 2009; Abdelatif et al., 2010) due to the decreased blood volume (Cork and Halliwell, 2002). Albumin plays an important role in osmoregulation and fluid movement control between different body compartments since it is a major contributor to blood colloid osmotic pressure; for this reason the rates of albumin breakdown and synthesis are regulated in response to dehydration to maintain normal colloid osmotic pressure and 
fluid distribution (Burton, 1988). Consequently some variations in serum albumin levels can occur, but the maintenance of normal levels has to be re-established as soon as amino acids from other sources like the skeletal muscle are available (Moorby et al., 2002). Alamer (2006) stated that serum hyperproteinemia is largely contributes in the maintainance of blood volume by inducing water movement in to the vascular system. The observed hyperalbuminemia could indicated an adjustable mechanism to maintain blood hydrostatic pressure and this response is mainly attributed to haemoconcentration (Ayoub and Saleh, 1998). Khan et al., (1978) reported nonsignificant increase in serum protein in Barmer goats deprived of water for 96 hours. Boyd (1981) also reported that plasma protein concentrations were increased by $14 \%, 62 \%$, $12 \%$ and $37 \%$ respectively in horse, sheep, cattle and donkey during thermal stress and water deprivation for 3-9 days. Ghosh et al., (1983) reported that there were increased protein levels in water deprived animals for 6 days. Gradual increase in serum albumin in Anglo-Nubian, Baladi and their crossbred goats to water deprivation for 3 days was noted by Hassan (1989). In contrast to the present study, reduction in total protein and albumin levels was noticed after 3 days of water deprivation in Awassi (Hamadeh et al., 2006) and Barki sheep under water stress (ElSherif and Assad, 2001) suggesting that low feed intake was the reason for this reduction. Again, albumin and protein levels tend to decrease after prolonged water restriction were reported by other studies (Hamadeh et al., 2006; Ghanem et al., 2008), which reflects dietary deficiency. Serum albumin serves as a labile protein reservoir providing a readily available source of amino acids until an alternative source is secured through diet or by mobilizing body sources such as skeletal muscle (Moorby et al., 2002).
Table 1 depicted that total serum protein and albumin concentrations were higher in water deprived animals compared to T2 and control animals. Lower serum protein and albumin concentrations were also reported in vitamin $\mathrm{C}$ administered water restricted Awassi as compared to non-supplemented counterparts (Ghanem, 2005), while others found no significant difference in total protein and globulin levels due to vitamin $\mathrm{C}$ supplementation (Hamadeh et al., 2009).

Overall summer values of total protein and albumin were higher in all the groups (Table $1)$. Significantly higher $(\mathrm{P}<0.05)$ protein level was found in control group whereas albumin levels were significantly higher $(\mathrm{P}<0.05)$ during summer in control and T1 groups. Similar findings were also observed in Beetal and Toggenberg goats (Kour et al., 2017). Higher protein and albumin concentrations during summer season might indicate a loss of extracellular fluid due to heat exposure, resulted in sharp increase in ADH level which was associated with a significant decrease in urine output and a significant increase in plasma protein. Higher concentration of albumin during summer season was also recorded by Ganaie et al., (2013) in cows and buffalo calves, in which they described that albumin is the major extracellular source of thiols, which are scavengers of free radicals allowing albumin to function as an antioxidant (Halliwel, 1996; Rasooli et al., 2004). Higher plasma total protein and albumin during summer content was recorded in other species (Koubkoba et al., 2002; Rasooli et al., 2004; Shrikhande et al., 2008). In contrast, no significant rise in the plasma protein concentration was found in summer in goat (Kataria et al., 1993; Sejian and Srivastava, 2009) and sheep (Adewuyi and Adu, 1983). Similarly some workers reported heat stress induced reduction in plasma albumin concentrations in other breeds of goat (Al-Eissa et al., 2012) and sheep 
(Abdoun et al., 2012). On the other hand, other scientists found that season had no significant effect on the concentration of total protein in Nubian goats (Abdelatif et al., 2010) and sheep (Hooda and Naqvi, 1990).

\section{Serum cholesterol}

As shown in the Table 1, the cholesterol level showed increasing trend from day 1 gradually up to day 22 in water deprived goats and all the values varied significantly $(\mathrm{P}<0.05)$ with each other in both seasons. In T1 group, the cholesterol values were ranged between 87.18 \pm 0.84 to $108.58 \pm 0.84 \mathrm{mg} / \mathrm{dl}$ and $87.87 \pm$ 1.26 to $108.23 \pm 1.29 \mathrm{mg} / \mathrm{dl}$ during summer and winter, respectively. Increase in blood cholesterol level upon water restriction of 20 and $40 \%$ was found by Casamassima et al., (2008) in lactating sheep. Adapted breeds under water restriction during hot and dry seasons mobilize their fat stores in order to overcome dietary deficiency (Chedid et al., 2014). As water intake is directly related to feed consumption (Silanikove, 1992), during water restriction feed consumption decreased, which is responsible for mobilization of reserve fat and increased cholesterol concentration in this group. Thando (2016) observed highest serum cholesterol concentration in goats deprived of water for 24 and 48 hours compared to those deprived of water for 0 hour $(\mathrm{P}<0.01)$.

Vitamin C supplemented group, cholesterol level was decreased gradually from day 7 up to 22 day during both seasons (Table 1). In this group, significantly lower $(\mathrm{P}<0.05)$ cholesterol levels were observed on 14 and 22 day compared to day 1 . Higher values were found on later stages of experiment (14 and 22 day) in $\mathrm{T} 1$ than the control value followed by $\mathrm{T} 2$ and the values were differ significantly $(\mathrm{P}<0.05)$ among the groups. Jaber et al., (2004) found that in the experimental sheep, which received water once every 4 days showed significantly $(\mathrm{P}<0.05)$ higher cholesterol concentration than the control group, which received water daily during the experimental period. Hamadeh et al., (2006) reported that ewes under water restriction showed higher serum cholesterol as compared to daily-watered animals. In contrast, other findings showed a tendency for higher cholesterol level under vitamin $\mathrm{C}$ supplementation (Ghanem, 2005; Ghanem et al., 2008; Karnib et al., 2009).

Overall summer and winter values were 87.50 $\pm 0.71 \mathrm{mg} / \mathrm{dl}$ and $86.48 \pm 0.43 \mathrm{mg} / \mathrm{dl}$ in control, $96.70 \pm 1.70 \mathrm{mg} / \mathrm{dl}$ and $96.55 \pm 1.68$ $\mathrm{mg} / \mathrm{dl}$ in $\mathrm{T} 1$ and $83.31 \pm 1.11 \mathrm{mg} / \mathrm{dl}$ and 85.35 $\pm 1.68 \mathrm{mg} / \mathrm{dl}$ in $\mathrm{T} 2$, respectively (Table 1); however, the values did not varied significantly. Kour et al., (2017) found that between summer, winter and autumn seasons, significantly higher $(\mathrm{P}<0.01)$ value was found in winter followed by autumn season. Cold environment could be a stimulus to augment thyroid hormone secretion to increase BMR in order to maintain body temperature which accompanies with high level of cholesterol (Prakash and Rathore, 1991; Kataria et al., 1993). Same findings were also observed in goat (Kataria et al., 1993), reindeer calves (Soveri et al., 1992), cattle (Rasooli et al., 2004) and camel (Kataria et al., 2002).

Pandey et al., (2012) showed that the concentration of cholesterol in goat was higher during hot season. On the other hand, some authors reported that in cattle and in neonatal calves, season had no effect on serum cholesterol level (Kweon et al., 1986; Chand and Georgie, 1989). The decreased level of cholesterol in T2 group during summer season may be due to decreased feed intake which was observed in other studies (Nazifi et al., 2003; Sejian et al., 2010; Ramana et al., 2013; Indu et al., 2014; Rathwa et al., 2017). 
Table.1 Biochemical profile (Mean \pm SE) in control and treatment groups of goats during summer and winter seasons

\begin{tabular}{|c|c|c|c|c|c|c|c|c|c|c|}
\hline Season & \multicolumn{5}{|c|}{ Summer } & \multicolumn{5}{|c|}{ Winter } \\
\hline Days & 1 & 7 & 14 & 22 & Total & 1 & 7 & 14 & 22 & Total \\
\hline \multicolumn{11}{|c|}{ Blood glucose (mg/ml) } \\
\hline Control & $\begin{array}{l}67.10 \\
\pm 0.50\end{array}$ & $\begin{array}{c}66.70^{\mathrm{B}} \\
\pm 0.55\end{array}$ & $\begin{array}{c}65.52^{\mathrm{B}} \\
\pm 0.58\end{array}$ & $\begin{array}{c}64.70^{B} \\
\pm 0.57\end{array}$ & $\begin{array}{c}66.00^{\mathrm{Bp}} \\
\pm 0.32\end{array}$ & $\begin{array}{l}69.15^{\mathrm{a}} \\
\pm 0.38\end{array}$ & $\begin{array}{c}69.27^{\mathrm{Ba}} \\
\pm 0.29\end{array}$ & $\begin{array}{c}70.17^{\mathrm{Cab}} \\
\pm 0.38\end{array}$ & $\begin{array}{c}71.47^{\mathrm{Cb}} \\
\pm 0.34\end{array}$ & $\begin{array}{c}70.01^{\mathrm{Cq}} \\
\pm 0.25\end{array}$ \\
\hline $\mathbf{T 1}$ & $\begin{array}{l}67.42^{\mathrm{a}} \\
\pm 1.00\end{array}$ & $\begin{array}{c}63.60^{\mathrm{Ac}} \\
\pm 0.82\end{array}$ & $\begin{array}{c}58.62^{\mathrm{Ab}} \\
\pm 0.65\end{array}$ & $\begin{array}{c}51.27^{\mathrm{Aa}} \\
\pm 0.38\end{array}$ & $\begin{array}{c}60.23^{\mathrm{A}} \\
\pm 1.31 \\
\end{array}$ & $\begin{array}{l}68.78^{\mathrm{d}} \\
\pm 0.72\end{array}$ & $\begin{array}{c}65.73^{\mathrm{Ac}} \\
\pm 0.47\end{array}$ & $\begin{array}{c}61.10^{\mathrm{Ab}} \\
\pm 0.50\end{array}$ & $\begin{array}{c}53.72^{\mathrm{Aa}} \\
\pm 0.28\end{array}$ & $\begin{array}{c}62.38^{\mathrm{A}} \\
\pm 1.19\end{array}$ \\
\hline $\mathbf{T 2}$ & $\begin{array}{l}67.75^{\mathrm{c}} \\
\pm 0.77\end{array}$ & $\begin{array}{c}65.93^{\mathrm{Bbc}} \\
\pm 0.71\end{array}$ & $\begin{array}{c}62.23^{\mathrm{Bab}} \\
\pm 0.69\end{array}$ & $\begin{array}{c}63.27^{\mathrm{Ba}} \\
\pm 0.69\end{array}$ & $\begin{array}{c}65.30^{\mathrm{Bp}} \\
\pm 0.49\end{array}$ & $\begin{array}{l}69.37^{b} \\
\pm 0.66\end{array}$ & $\begin{array}{c}68.25^{\mathrm{Bb}} \\
\pm 0.70\end{array}$ & $\begin{array}{c}67.11^{\mathrm{Bb}} \\
\pm 0.63\end{array}$ & $\begin{array}{c}64.05^{\mathrm{Ba}} \\
\pm 0.79\end{array}$ & $\begin{array}{c}67.20^{\mathrm{Bq}} \\
\pm 0.53\end{array}$ \\
\hline \multicolumn{11}{|c|}{ Total protein (gm/dl) } \\
\hline Control & $\begin{array}{c}6.39 \\
\pm 0.06\end{array}$ & $\begin{array}{l}6.42^{\mathrm{A}} \\
\pm 0.06\end{array}$ & $\begin{array}{l}6.43^{\mathrm{A}} \\
\pm 0.06\end{array}$ & $\begin{array}{l}6.54^{\mathrm{A}} \\
\pm 0.07\end{array}$ & $\begin{array}{l}6.44^{\mathrm{Aq}} \\
\pm 0.03\end{array}$ & $\begin{array}{c}6.32 \\
\pm 0.03\end{array}$ & $\begin{array}{l}6.30^{\mathrm{A}} \\
\pm 0.23\end{array}$ & $\begin{array}{l}6.29^{\mathrm{A}} \\
\pm 0.02\end{array}$ & $\begin{array}{l}6.28^{\mathrm{A}} \\
\pm 0.03\end{array}$ & $\begin{array}{l}6.30^{\mathrm{Ap}} \\
\pm 0.01\end{array}$ \\
\hline T1 & $\begin{array}{l}6.43^{\mathrm{a}} \\
\pm 0.06\end{array}$ & $\begin{array}{l}6.63^{\mathrm{Bb}} \\
\pm 0.05\end{array}$ & $\begin{array}{l}7.01^{\mathrm{Cc}} \\
\pm 0.07\end{array}$ & $\begin{array}{l}7.43^{\mathrm{Cd}} \\
\pm 0.06\end{array}$ & $\begin{array}{l}6.88^{\mathrm{C}} \\
\pm 0.09\end{array}$ & $\begin{array}{r}6.36^{\mathrm{a}} \\
\pm 0.04\end{array}$ & $\begin{array}{l}6.60^{\mathrm{Bb}} \\
\pm 0.06\end{array}$ & $\begin{array}{l}6.84^{\mathrm{Cc}} \\
\pm 0.03\end{array}$ & $\begin{array}{l}7.25^{\mathrm{Cd}} \\
\pm 0.03\end{array}$ & $\begin{array}{l}6.76^{\mathrm{C}} \\
\pm 0.07\end{array}$ \\
\hline $\mathbf{T} 2$ & $\begin{array}{l}6.45^{\mathrm{a}} \\
\pm 0.02\end{array}$ & $\begin{array}{c}6.49^{\mathrm{ABab}} \\
\pm 0.02\end{array}$ & $\begin{array}{c}6.66^{\mathrm{Bbc}} \\
\pm 0.04\end{array}$ & $\begin{array}{l}6.82^{\mathrm{Bc}} \\
\pm 0.03\end{array}$ & $\begin{array}{l}6.60^{\mathrm{B}} \\
\pm 0.03\end{array}$ & $\begin{array}{c}6.36^{\mathrm{a}} \\
\pm 0.03\end{array}$ & $\begin{array}{l}6.51^{\mathrm{Bb}} \\
\pm 0.04\end{array}$ & $\begin{array}{l}6.64^{\mathrm{Bc}} \\
\pm 0.02\end{array}$ & $\begin{array}{l}6.77^{\mathrm{Bd}} \\
\pm 0.02\end{array}$ & $\begin{array}{l}6.57^{\mathrm{B}} \\
\pm 0.03\end{array}$ \\
\hline \multicolumn{11}{|c|}{ Albumin (gm/dl) } \\
\hline Control & $\begin{array}{l}2.57 \\
\pm 0.09\end{array}$ & $\begin{array}{l}2.53^{\mathrm{A}} \\
\pm 0.06\end{array}$ & $\begin{array}{l}2.67^{\mathrm{A}} \\
\pm 0.06\end{array}$ & $\begin{array}{l}2.75^{\mathrm{A}} \\
\pm 0.04\end{array}$ & $\begin{array}{l}2.63^{\mathrm{Aq}} \\
\pm 0.04\end{array}$ & $\begin{array}{c}2.55 \\
\pm 0.07\end{array}$ & $\begin{array}{c}2.59 \\
\pm 0.07\end{array}$ & $\begin{array}{l}2.52^{\mathrm{A}} \\
\pm 0.05\end{array}$ & $\begin{array}{l}2.40^{\mathrm{A}} \\
\pm 0.04\end{array}$ & $\begin{array}{l}2.51^{\mathrm{Ap}} \\
\pm 0.03\end{array}$ \\
\hline T1 & $\begin{array}{l}2.63^{\mathrm{a}} \\
\pm 0.06\end{array}$ & $\begin{array}{c}2.93^{\mathrm{Bb}} \\
\pm 0.10\end{array}$ & $\begin{array}{l}3.43^{\mathrm{Cc}} \\
\pm 0.07\end{array}$ & $\begin{array}{l}4.11^{\mathrm{Cc}} \\
\pm 0.14\end{array}$ & $\begin{array}{l}3.28^{\mathrm{Cq}} \\
\pm 0.13\end{array}$ & $\begin{array}{r}2.66^{\mathrm{a}} \\
\pm 0.09\end{array}$ & $\begin{array}{l}2.83^{\mathrm{ab}} \\
\pm 0.08\end{array}$ & $\begin{array}{l}3.07^{\mathrm{Bb}} \\
\pm 0.09\end{array}$ & $\begin{array}{l}3.40^{\mathrm{Bc}} \\
\pm 0.06\end{array}$ & $\begin{array}{l}2.99^{\mathrm{Bp}} \\
\pm 0.07\end{array}$ \\
\hline $\mathbf{T} 2$ & $\begin{array}{l}2.61^{\mathrm{a}} \\
\pm 0.06\end{array}$ & $\begin{array}{c}2.77^{\mathrm{ABab}} \\
\pm 0.03\end{array}$ & $\begin{array}{c}3.03^{\mathrm{Bbc}} \\
\pm 0.05\end{array}$ & $\begin{array}{l}3.17^{\mathrm{Bc}} \\
\pm 0.62\end{array}$ & $\begin{array}{l}2.90^{\mathrm{Bc}} \\
\pm 0.05\end{array}$ & $\begin{array}{c}2.57^{\mathrm{a}} \\
\pm 0.10\end{array}$ & $\begin{array}{l}2.73^{\mathrm{ab}} \\
\pm 0.11\end{array}$ & $\begin{array}{c}2.95^{\mathrm{Aab}} \\
\pm 0.12\end{array}$ & $\begin{array}{l}3.10^{\mathrm{Ab}} \\
\pm 0.15\end{array}$ & $\begin{array}{l}2.84^{\mathrm{B}} \\
\pm 0.07\end{array}$ \\
\hline \multicolumn{11}{|c|}{ cholesterol (mg/dl) } \\
\hline Control & $\begin{array}{l}87.83 \\
\pm 1.63\end{array}$ & $\begin{array}{c}87.92^{\mathrm{AB}} \\
\pm 1.33\end{array}$ & $\begin{array}{c}86.93^{\mathrm{B}} \\
\pm 1.23\end{array}$ & $\begin{array}{c}87.32^{\mathrm{B}} \\
\pm 1.80\end{array}$ & $\begin{array}{c}87.50^{\mathrm{B}} \\
\pm 0.71\end{array}$ & $\begin{array}{l}86.93 \\
\pm 0.96\end{array}$ & $\begin{array}{c}86.67^{\mathrm{A}} \\
\pm 0.89\end{array}$ & $\begin{array}{c}86.75^{\mathrm{A}} \\
\pm 0.90\end{array}$ & $\begin{array}{c}85.58^{\mathrm{A}} \\
\pm 0.78\end{array}$ & $\begin{array}{c}86.48^{\mathrm{A}} \\
\pm 0.43\end{array}$ \\
\hline
\end{tabular}




\begin{tabular}{|c|c|c|c|c|c|c|c|c|c|c|}
\hline T1 & $\begin{array}{l}87.18^{\mathrm{a}} \\
\pm 0.84\end{array}$ & $\begin{array}{l}92.45^{\mathrm{Bb}} \\
\pm 0.900\end{array}$ & $\begin{array}{c}98.57^{\mathrm{Cc}} \\
\pm 0.55\end{array}$ & $\begin{array}{c}108.58^{\mathrm{Cd}} \\
\pm 0.84\end{array}$ & $\begin{array}{c}96.70^{\mathrm{C}} \\
\pm 1.70\end{array}$ & $\begin{array}{l}87.87^{\mathrm{a}} \\
\pm 1.26\end{array}$ & $\begin{array}{c}93.00^{\mathrm{Bab}} \\
\pm 1.32\end{array}$ & $\begin{array}{c}97.12^{\mathrm{Bb}} \\
\pm 1.48\end{array}$ & $\begin{array}{c}108.23^{\mathrm{Bc}} \\
\pm 1.29\end{array}$ & $\begin{array}{c}96.55^{\mathrm{B}} \\
\pm 1.68\end{array}$ \\
\hline $\mathbf{T} 2$ & $\begin{array}{l}87.82^{\mathrm{b}} \\
\pm 1.85\end{array}$ & $\begin{array}{c}85.93^{\text {Aab }} \\
\pm 1.50\end{array}$ & $\begin{array}{c}81.97^{\mathrm{Aa}} \\
\pm 1.41\end{array}$ & $\begin{array}{c}77.53^{\mathrm{Aa}} \\
\pm 1.57\end{array}$ & $\begin{array}{c}83.31^{\mathrm{A}} \\
\pm 1.11\end{array}$ & $\begin{array}{l}87.51 \\
\pm 1.20\end{array}$ & $\begin{array}{c}86.33^{\mathrm{A}} \\
\pm 1.20\end{array}$ & $\begin{array}{c}84.57^{\mathrm{A}} \\
\pm 1.26\end{array}$ & $\begin{array}{c}82.98^{\mathrm{A}} \\
\pm 1.40\end{array}$ & $\begin{array}{c}85.35^{\mathrm{A}} \\
\pm 1.68\end{array}$ \\
\hline \multicolumn{11}{|c|}{ urea (mg/dl) } \\
\hline Control & $\begin{array}{l}16.46 \\
\pm 0.72\end{array}$ & $\begin{array}{l}16.76^{\mathrm{A}} \\
\pm 0.74\end{array}$ & $\begin{array}{c}17.19^{\mathrm{A}} \\
\pm 0.72\end{array}$ & $\begin{array}{l}16.63^{\mathrm{A}} \\
\pm 0.62\end{array}$ & $\begin{array}{c}16.76^{\mathrm{Aq}} \\
\pm 0.33\end{array}$ & $\begin{array}{l}11.78 \\
\pm 0.40\end{array}$ & $\begin{array}{c}11.63^{\mathrm{A}} \\
\pm 0.39\end{array}$ & $\begin{array}{l}12.04^{\mathrm{A}} \\
\pm 0.43\end{array}$ & $\begin{array}{l}11.92^{\mathrm{A}} \\
\pm 0.36\end{array}$ & $\begin{array}{c}11.84^{A p} \\
\pm 0.19\end{array}$ \\
\hline T1 & $\begin{array}{l}17.09^{\mathrm{a}} \\
\pm 0.80\end{array}$ & $\begin{array}{c}20.06^{\mathrm{Bb}} \\
\pm 0.65\end{array}$ & $\begin{array}{c}23.72^{\mathrm{Bc}} \\
\pm 0.36\end{array}$ & $\begin{array}{c}28.11^{\mathrm{Bd}} \\
\pm 0.40\end{array}$ & $\begin{array}{c}22.24^{\mathrm{Bq}} \\
\pm 0.90\end{array}$ & $\begin{array}{l}12.03^{\mathrm{a}} \\
\pm 0.48\end{array}$ & $\begin{array}{c}14.02^{\mathrm{Ba}} \\
\pm 0.48\end{array}$ & $\begin{array}{c}18.27^{\mathrm{Bb}} \\
\pm 0.86\end{array}$ & $\begin{array}{c}23.95^{\mathrm{Bc}} \\
\pm 0.89\end{array}$ & $\begin{array}{c}17.07^{\mathrm{Bp}} \\
\pm 1.01\end{array}$ \\
\hline $\mathbf{T} 2$ & $\begin{array}{l}16.79 \\
\pm 0.84\end{array}$ & $\begin{array}{c}17.73^{\mathrm{A}} \\
\pm 0.70\end{array}$ & $\begin{array}{l}18.29^{\mathrm{B}} \\
\pm 0.62\end{array}$ & $\begin{array}{l}18.19^{\mathrm{A}} \\
\pm 0.53\end{array}$ & $\begin{array}{c}17.74^{\mathrm{Aq}} \\
\pm 0.34\end{array}$ & $\begin{array}{l}11.90 \\
\pm 0.35\end{array}$ & $\begin{array}{c}12.51^{\mathrm{AB}} \\
\pm 0.40\end{array}$ & $\begin{array}{c}12.76^{\mathrm{A}} \\
\pm 0.59\end{array}$ & $\begin{array}{c}12.94^{\mathrm{A}} \\
\pm 0.63\end{array}$ & $\begin{array}{c}12.53^{\mathrm{Ap}} \\
\pm 0.25\end{array}$ \\
\hline \multicolumn{11}{|c|}{ creatinine (mg/dl) } \\
\hline Control & $\begin{array}{c}0.61 \\
\pm 0.03\end{array}$ & $\begin{array}{c}0.62 \\
\pm 0.03\end{array}$ & $\begin{array}{l}0.61^{\mathrm{A}} \\
\pm 0.02\end{array}$ & $\begin{array}{l}0.63^{A} \\
\pm 0.02\end{array}$ & $\begin{array}{l}0.61^{\mathrm{A}} \\
\pm 0.01\end{array}$ & $\begin{array}{c}0.63 \\
\pm 0.03\end{array}$ & $\begin{array}{c}0.64 \\
\pm 0.04\end{array}$ & $\begin{array}{l}0.66^{\mathrm{A}} \\
\pm 0.04\end{array}$ & $\begin{array}{l}0.63^{\mathrm{A}} \\
\pm 0.02\end{array}$ & $\begin{array}{l}0.64^{\mathrm{A}} \\
\pm 0.02\end{array}$ \\
\hline $\mathbf{T 1}$ & $\begin{array}{l}0.61^{\mathrm{a}} \\
\pm 0.42\end{array}$ & $\begin{array}{l}0.71^{\mathrm{ab}} \\
\pm 0.04\end{array}$ & $\begin{array}{c}0.72^{\mathrm{Bbc}} \\
\pm 0.03\end{array}$ & $\begin{array}{l}1.04^{\mathrm{Bc}} \\
\pm 0.20\end{array}$ & $\begin{array}{l}0.80^{\mathrm{B}} \\
\pm 0.06\end{array}$ & $\begin{array}{c}0.59^{\mathrm{a}} \\
\pm 0.03\end{array}$ & $\begin{array}{c}0.66^{\mathrm{a}} \\
\pm 0.03\end{array}$ & $\begin{array}{l}0.79^{\mathrm{Bb}} \\
\pm 0.02\end{array}$ & $\begin{array}{l}0.89^{\mathrm{Cc}} \\
\pm 0.02\end{array}$ & $\begin{array}{l}0.73^{\mathrm{B}} \\
\pm 0.03\end{array}$ \\
\hline $\mathbf{T 2}$ & $\begin{array}{c}0.60 \\
\pm 0.03\end{array}$ & $\begin{array}{c}0.65 \\
\pm 0.03\end{array}$ & $\begin{array}{c}0.69^{\mathrm{AB}} \\
\pm 0.03\end{array}$ & $\begin{array}{l}0.72^{\mathrm{A}} \\
\pm 0.03\end{array}$ & $\begin{array}{l}0.67^{\mathrm{A}} \\
\pm 0.03\end{array}$ & $\begin{array}{l}0.60^{\mathrm{a}} \\
\pm 0.04\end{array}$ & $\begin{array}{l}0.65^{\mathrm{ab}} \\
\pm 0.03\end{array}$ & $\begin{array}{c}0.69^{\mathrm{ABab}} \\
\pm 0.03\end{array}$ & $\begin{array}{l}0.73^{\mathrm{Bb}} \\
\pm 0.03\end{array}$ & $\begin{array}{c}0.68^{\mathrm{AB}} \\
\pm 0.02\end{array}$ \\
\hline
\end{tabular}

$\mathrm{a}, \mathrm{b}, \mathrm{c}, \mathrm{d}$ describe significant differences within groups $(\mathrm{P}<0.05)$

A, B, C describe significant differences between groups $(\mathrm{P}<0.05)$

$\mathrm{p}, \mathrm{q}$ describe significant differences between seasons $(\mathrm{P}<0.05)$ 


\section{Serum urea and serum creatinine}

Table 1 depicted that serum urea and creatinine levels were showed an increasing trend in water deprived goats during summer and winter seasons. The recorded values of urea in T1 group ranged from $17.09 \pm 0.80$ $\mathrm{mg} / \mathrm{dl}$ to $28.11 \pm 0.40 \mathrm{mg} / \mathrm{dl}$ in summer and $12.03 \pm 0.48 \mathrm{mg} / \mathrm{dl}$ to $23.95 \pm 0.89 \mathrm{mg} / \mathrm{dl}$ in winter; the creatinine values ranged from 0.61 $\pm 0.42 \mathrm{mg} / \mathrm{dl}$ to $1.01 \pm 0.20 \mathrm{mg} / \mathrm{dl}$ and $0.59 \pm$ $0.03 \mathrm{mg} / \mathrm{dl}$ to $0.89 \pm 0.02 \mathrm{mg} / \mathrm{dl}$ in summer and winter, respectively. Water stress causes a decrease in urine output and the production of dry faeces due to increased water reabsorption from the gastro-intestinal tract (Olsson et al., 1982). During water stress, the transfer function of the kidney is altered (Kataria and Kataria, 2007) with slower glomerular filtration rate and higher urea reabsorption (Silanikove, 2000) and consequently the levels of urea and creatinine in blood were increased. Similar observations were also reported by other authors (Laden et al., 1987; Igbokwe, 1993; Jaber et al., 2004). Hamadehet al., (2006) and Alamer (2005) also observed an increase in this parameter in water restricted animals. However, upon prolonged water restriction and reduced feed intake, urea levels may start to decline reflecting an increase in urea recycling into the gut (Igbokwe, 1993; Marini et al., 2004), so it can be used as a nitrogen source by rumen microflora. In contrast, creatinine levels in lambs were not affected by 48 hours of water restriction (Jacob et al., 2006). The creatinine concentration was influenced by the level of proteolysis and endogenous nitrogen sources (Caldeira et al., 2007; Kataria and Kataria, 2007) as well as by higher kidney retention due to decreased GFR.

The urea level showed increasing trend from day 1 gradually to day 14, then decreased on day 22 in vitamin supplemented group during summer (Table 1). Our study also revealed that urea and creatinine values were significantly lower $(\mathrm{P}<0.05)$ in vitamin supplementation group than water deprived goats. With the supplementation of vitamin $\mathrm{C}$ in water deprived goats (T2) the levels comes to normal range, might be due to beneficial effect of vitamin $\mathrm{C}$ supplementation. In another experiment (Hamadeh et al., 2009), the daily dose of $5 \mathrm{gm}$ of vitamin $\mathrm{C}$ significantly increased serum creatinine and urea concentrations as compared to $3 \mathrm{gm}$ and control in water restricted Awassi sheep. On the other hand, Karnib (2009) showed no effect of vitamin $\mathrm{C}$ supplementation on creatinine level during dehydration. Zetingrao (2003) observed that in early summer significantly increased $(\mathrm{P}<0.01)$ serum urea was recorded in deprived animals (27.62 \pm $8.90 \mathrm{mg} / \mathrm{dl})$ over the control animals $(15.04 \pm$ $4.93 \mathrm{mg} / \mathrm{dl}$ ) at only 24 hours of water deprivation. Boyd (1981) reported that in different species the serum urea level was elevated with dehydration. Ghosh et al., (1983) noted effect of short term water deprivation in Black Bengal goats; the serum urea in control group was $40.50 \pm 2.21 \mathrm{mg} / \mathrm{dl}$, in $50 \%$ deprived $36.75 \pm 10.75 \mathrm{mg} / \mathrm{dl}$ and $100 \%$ deprived group $45.74 \pm 3.60 \mathrm{mg} / \mathrm{dl}$ which indicated increase in value in fully deprived animals. Whereas, Igbokwe (1997) reported plasma urea concentration increased after short term water deprivation in sheep, goats and cattle but after prolonged water deprivation, plasma urea concentration returned to normal or decreased. Zetingrao (2003) indicated that serum urea returned close to normal values as summer stress was over; they also reported that in mid-summer, serum creatinine was significantly higher $(\mathrm{P}<0.05) \quad(1.23 \pm 0.13 \mathrm{mg} / \mathrm{dl}) \quad$ in deprived animals than in control animals $(1.11 \pm 0.13$ $\mathrm{mg} / \mathrm{dl}$ ) at 60 hours of deprivation; again highly significant increase $(\mathrm{P}<0.01)$ in serum creatinine in control $(1.05 \pm 0.04 \mathrm{mg} / \mathrm{dl})$ and in deprived animals $(1.25 \pm 0.05 \mathrm{mg} / \mathrm{dl})$ at 72 
hours of deprivation was noted. Boyd (1981) reported increase in serum creatinine concentration in different species due to effect of thermal stress and dehydration. Insignificant increase in serum creatinine concentration after six days deprivation in black Bengal goats was observed and the values recorded in control, $50 \%$ deprived and $100 \%$ deprived animals were $1.19 \pm 0.17$, $2.18 \pm 0.24,2.64 \pm 0.21 \mathrm{mg} / \mathrm{dl}$, respectively as reported by Ghosh et al., (1983). Plasma creatinine concentration has increased after short term water deprivation, but when deprivation was prolonged, the increase in plasma creatinine concentration was still sustained in different species (Igbokwe, 1997).

Table 1 depicted that significantly higher $(\mathrm{P}<0.05)$ urea level in summer in all groups; however no significant seasonal variation was observed in creatinine levels, in $\mathrm{T} 1$ group higher creatinine concentration was found during summer season. The high concentration of blood urea in summer months probably as a result of increase in loss of extra-cellular fluid due to heat exposure (Rasooli et al., 2004). Again, increased level of urea during heat exposure might be due to increased utilization of amino acids through mobilization of protein from muscle (Sreedhar et al., 2013). The increased level of these parameters might be due to reduced blood flow toward kidney during heat stress condition. Similar findings of increased blood urea and creatinine during summer season have been reported by other workers (Srikandakumar et al., 2003, Suhair, 2012, Ghosh et al., 2013; Indu et al., 2014). In contrast, others (Eldon et al., 1988; Shrikhande et al., 2008) found that BUN level did not differ significantly during summer and winter, but was significantly low during rainy season (10.86 mg/dl). Rathwa et al., (2017) observed that the level of BUN and creatinine was increased significantly $(\mathrm{P}<0.05)$ in summer season.

\section{References}

Abdelatif, A. M., Elsayed, S. A. and Hassan, Y. M. 2010. Effect of state of hydration on body weight, blood constituents and urine excretion in Nubian goats (Capra hircus). World Journal of Agricultural Sciences, 6(2): 178-188.

Abdoun, K. A., Samara, E. M., Okab, A. B. and Al-Haidary, A. A. 2012. Regional and circadian variations of sweating rate and body surface temperature in camels (Camelus dromedarius). Animal Science Journal, 83: 556-561.

Adewuyi, A. A. and Adu, I. F. 1983. Seasonal variation in the levels of some blood components of indigenous and crossbred sheep. Tropical Animal Health and Production, 9: 223- 230.

Alamer, M. 2005. Effect of deprivation and season on some biochemical constituents of blood in Awassi and Najdi sheep breeds in Saudi Arabia. Journal of Animal and Veterinary Advances, 48: 15-20.

Alamer, M. 2006. Physiological responses of Saudi Arabia indigenous goats to water deprivation. Small Ruminant Research, 63(1-2): 100-109.

Al-Eissa, M. S., Alkahtani, S., Al-Farraj, S. A., Alarifi, S. A., Al-Dahmash, B. and AlYahya, H. 2012. Seasonal variation effects on the composition of blood in Nubian ibex (Capra nubiana) in Saudi Arabia. African Journal of Biotechnology, 11(5): 1283-1286.

Ayoub, M.A. and Saleh, A.A. 1998. A comparative physiological study between camels and goats during water deprivation. Proceedings of the Third Annual Meeting for Animal Production Under Arid Conditions, 1:71-87, United Arab Emirates University.

Banerjee, D., Upadhyay, R. C., Chaudhary, U. B., Kumar, R., Singh, S., Ashutosh, D. T. K. and De, S. 2015. Seasonal variations in physio-biochemical profiles of Indian goats in the paradigm of hot and cold climate. Biological Rhythm Research, 
46(2): 221-236.

Boyd, C. E. 1981. Water Quality in warm water fish ponds. Anburn University, Alabama. 359p. Craftmaster Printers, Inc. Opelika, Alabama.

Burstein, M., Scholnick, H. R. and Morfin, R. 1970. Rapid method for the isolation of lipoprotein from human serum by precipitation with polyanions. Journal of Lipid Research, 11: 583-595.

Burton, R. F. 1988. The protein content of extracellular fluids and its relevance to the study of ionic regulation: net charge and colloid osmotic pressure. Comparative Biochemistry and Physiology, 90A: 11-16

Caldeira, A., Belo, C., Santos, M., Vazques, A. and Portugal, A. V. 2007. The effect of body condition score on blood metabolites and hormonal profiles in ewes. Small Ruminant Research, 68(3): 233-241.

Casamassima, D., Pizzo, R., Palazzo, M., D' Alessandro, A. G. and Martemucci, G. 2008. Effect of water restriction on productive performance and blood parameters in Comisana sheep reared under intensive condition. Small Ruminant Research, 78: 169-175.

Chand, D. and Georgie, G. C. 1989. Influence of season and genetic group on the blood plasma cholesterol in neonate calves. Indian Journal of Animal Sciences,59: 149-153.

Chedid, M., Lina, S., Giger-Reverdin, J. S., Duvaux-Ponter, $\mathrm{C}$ and Hamadeh, S. K. 2014. Review: Water stress in sheep raised under arid conditions. Canadian Journal of Animal Science, 94: 243-257.

Collier, R. J., Collier, J. L., Rhoads, R. P. and Baumgard, L. H. 2008. Gene involved in the bovine heat stress response. Journal of Dairy Science, 91: 445-454.

Cork, S. C. and Halliwell, R. W. 2002. The veterinary laboratory and field manual. Nottingham University Press, Nottingham, UK

Doumasa, B. T., Arendis, R. L. and Pinto, P. C. 1971. Standard Method of Clinical Chemistry, pp. 175-189. Academic Press, Chicago.
Eldon, J., Thorsteinsson, Th. and Olafsson, Th. 1988. The concentration of blood glucose, urea, calcium and magnesium in milking dairy cows. Journal of Veterinary Medicine Series A, 35(1-10): 44-53.

El-Sherif, M. M. A. and Assad, F. 2001. Changes in some blood constituents in Barki ewes during pregnancy and lactation under semi-arid conditions. Small Ruminant Research, 40: 269-277.

Ganaie, A. H., Ghasura, R. S., Mir, N. A., Bumla, N. A., Sankar, G. and Wani, S. A., 2013. Biochemical and physiological changes during thermal stress in bovines: A Review. Iranian Journal of Applied Animal Science, 3(3): 423-430.

Ghanem, A. M., Barbour, E. K., Hamadeh, S. K., Jaber, L. S. and Abi Said, M. 2008. Physiological and chemical responses in water-deprived Awassi ewes treated with vitamin C. Journal of Arid Environments, 72(3): 141-149.

Ghanem, A. M., Barbour, E. K., Hamadeh, S. K., Jaber, L. S. and Abi Said, M. 2008. Physiological and chemical responses in water-deprived Awassi ewes treated with vitamin C. Journal of Arid Environments, 72(3): 141-149.

Ghanem A. 2005. The effect of vitamin C supplementation on some physiological an immunological indicators in waterdeprived Awassi ewes. Master thesis. American University of Beirut, p:1-89.

Ghosh, S., Singh, A. K. and Haldar, C. 2013. Adaptive and ecological significance of the seasonal changes in hematological, biochemical and hormonal parameters in the tropical goat (Capra hircus). Journal of Endocrinology and Reproduction, 17(2): 113-122.

Ghosh, T. K., Bhattacharyya, B. and Moitra, D. N. 1983. Effect of short term water deprivation on physiological performance and certain bloo constituents in black Bengal goats. Indian Veterinary Journal, 60: 969-972.

Gomall, A. J. 1949. Determination of serum proteins by means of the Biuret reaction. Biological Chemistry, 177: 751. 
Grunwaldt, E. G., Guevara, J. C., Estevez, O. R., Vicente, A., Rousselle, H., Alcuten, N., Aguerregaray, D. and Stasi, C. R. 2005. Biochemical and Haematological Measurements in Beef Cattle in Mendoza Plain Rangelands (Argentina). Tropical Animal Health and Production, 37(6): 527-540.

Halliwell, B. 1996. Antioxidants in human health and disease. Annual Review of Nutrition, 16: 33-50.

Hamadeh, S. K., Rawda, N., Jaber, L. S., Habre, A., Abi Said, M. and Barbour, E. K. 2006. Physiological responses to water restriction in dry and lactating Awassi ewes. Livestock Science, 101(1-3): 101109.

Hamadeh, S. K., Hanna, N., Barbour, E. K., Abi Said, M., Rawda, N., Chedid, M. and Jaber, L. S. 2009. Changes in physiological and blood parameters in water restricted Awassi ewes supplemented with different levels of Vitamin C. In European Federation for Animal Science EAAP, 60th Annual Meeting, Barcelona, 27 August. Session S26 Abstract no. 3175

Hassan, G. A. E. 1989. Physiological responses of Anglo-Nubian and Baladi goats and their crossbreds to water deprivation under sub-tropical conditions. Livestock Production Science, 22: 295-304.

Hooda, O. K. and Naqvi, S. M. K. 1990. Effect of thermal load and feed restriction on relative adaptability of Malpura and Avikalin sheep in semi-arid region. Indian Journal of Animal Science, 60: 608-611.

Igbokwe, I. O. 1997. The effects of water deprivation in livestock ruminants: an overview. Nutritional Abstracts and Review (Series B), 67: 905-914.

Igbokwe, I. O. 1993. Haemoconcentration in Yankasa sheep exposed to prolonged water deprivation. Small Ruminant Research, 12: 99-105.

Indu, S., Sejian, V. and Naqvi, S. M. K. 2014. Impact of stimulated heat stress on growth, physiological adaptability, blood metabolites and endocrine responses in
Malpura ewes under semiarid tropical environment. Animal Production Science, 55(6): 766-776.

Itoh, F., Obara, Y., Rose, M. T., Fuse, H. and H. Hashimoto. 1998. Insulin and glucagon secretion in lactating cows during heat exposure. Journal of Animal Sciences, 76: 2182-2189.

Jaber, L. S., Habre, A., Rawda, N., Said, M. A., Barbour, E. K. and Hamadeh, S. 2004. The effect of water restriction on certain physiological parameters in Awassi sheep. Small Ruminant Research, 54(1-2): 115120.

Jacob, R. H., Pethick, D. W., Clark, P., D'Souza, D. N., Hopkins, D. L and White, J. 2006. Quantifying the hydration status of lambs in relation to carcass characteristics. Australian Journal of Experimental Agriculture, 46(4): 429-437.

Karnib, M. 2009. The Effect of Vitamin C Administration on some Physiological Parameters in Water and Feed Restricted Dry Awassi Ewes. Master Thesis, American University of Beirut; pp.1-48.

Kataria, N. and Kataria, A. K. 2007. Compartmental water management of Marwari sheep. Veterinarski Arhiv, 77(6): 551-559.

Kataria, A. K., Kataria, N., Bhatia, J. S. and Ghosal, A. K. 1993. Blood metabolic profile of Marwari goats in relation to seasons. Indian Veterinary Journal,70: 761-762.

Kataria, N., Kataria, A. K., Agarwal, V. K., Garg, S. L. and Sahani, M. S. 2002. Effect of long term dehydration on serum constituents in extreme climatic conditions in camel. Indian Journal of Physiology and Pharmacology, 46: 218222.

Khan, M. S., Ghosh, P. K. and Sasidharan, T. O. 1978. Effect of acute water restriction on plasma proteins and on blood and urinary electrolytes in Barmer goats of the Rajasthan desert. The Journal of Agricultural Science, 91(2): 395-398.

Koubkova, M., Knizkova, I., Kunc, P., Hartlova, H., Flusser, J. and Dolezal, O. 
2002. Influence of high environmental temperatures and evaporative cooling on some physiological, haematological and biochemical parameters in high-yielding dairy cows. Czech Journal of Animal Science, 47: 309-318.

Kour, S., Devi, J., Kour, K., Chakraborty, D., Ganai, A. W., Zargar, R. and Khajuria, P. 2017. Effect of Extreme Seasons on Some Blood Biochemical Parameters in Beetal and Toggenberg Goats of Jammu Region. Journal of Animal Research. 7(4): 779784.

Kour, S., Devi, J., Kour, K., Chakraborty, D., Khajuria, P. and Zargar, R. 2015. Effect of Thermal Stress on Haematological Parameters and Enzymatic Activities in Two Breeds of Goat after Thermal Stress. Journal of Animal Research, 5(4): 855862. Kweon et al., 1986.

Laden, S., Nehmadi, L. and Yagil, R. 1987. Dehydration tolerance in Awassi fat-tailed sheep. Canadian Journal of Zoology, 65: 363-367.

Madziga, I. I., Alawa, C. B. I., Lamidi, O. S. and Goska, D. Y. 2013. Assessing the nutritional status of four indigenous breeds of cattle using some blood metabolites in Nigeria. Journal of Biology, Agriculture and Healthcare, 3(2): 1-8.

Marini, J. C., Klein, J. D., Sands, J. M. and Van Amburgh, M. E. 2004. Effect of nitrogen intake on nitrogen recycling and urea transporter abundance in lambs. Journal of Animal Science, 82: 1157-1164.

Moorby, J. M., Dewhurst, R. J., Evans, R. T and Fisher, W. J. 2002. Effects of level of concentrate feeding during the second gestation of Holstein-Friesian dairy cows. 2. Nitrogen balance and plasma metabolites. Journal of Dairy Science, 85: 178-189.

Nazifi, S., Saeb, M., Rowghani, E. and Kaveh, K. 2003. The influences of thermal stress on serum biochemical parameters of Iranian fat-tailed sheep and their correlation with triiodothyronine $\left(\mathrm{T}_{3}\right)$, thyroxine $\left(\mathrm{T}_{4}\right)$ and cortisol concentrations. Comparative Clinical Pathology,12(3):
135-139.

Ndlovu, T., Chimonyo, M., Okoh, A.I., Muchenje, V., Dzama, K. and Raats, J. G. 2007. Assessing the nutritional status of beef cattle: current practices and future prospects. African Journal Biotechnology, 6: 2727-2734.

Olsson K., Benlamlih S., Dahlborn K. and Fyhrqvist, F. 1982. Effects of water deprivation and hyperhydration in pregnant and lactating goats. Acta Physiologica Scandinavica, 15: 361-367.

Pandey, N., Kataria, N., Kataria, A. K. and Joshi, A. 2012. Ambient stress associated variation in metabolic responses of Marwari goat of arid tracts in India. Journal of Stress Physiology and Biochemistry, 8(3): 120-127.

Prakash, P. and Rathore, V. S. 1991. Seasonal Variations in blood serum profiles of triido- thyronine and thyroxine in goats. Indian Journal of Animal Science,61: 1311-1312.

Ramana, D. B. V., Pankaj, P. K., Nikhila, M., Rani, R. and Sudheer, D. 2013 Productivity \& physiological responses of sheep exposed to heat stress. Journal of Agrometeorology, 15: 71-76.

Rasooli, A., Nouri, M., Khadjeh, G. H. and Rasekh, A. 2004. The influences of seasonal variation on thyroid activity and some biochemical parameters of cattle. Iranian Journal of Veterinary Research,5: 1383-2004.

Rathwa, S. D., Vasava, A. A., Pathan, M. M., Madhira, S. P., Patel, Y. G. and Pande, A. M. 2017. Effect of season on physiological, biochemical, hormonal, and oxidative stress parameters of indigenous sheep. Veterinary World, 10: 650-654.

Roeschlau, P., Bernt, E. and Gruber, W. 1974. Enzymatic determination of total cholesterol in serum. Zeitschrift für klinische Chemie und klinische Biochemie, 12(5): 226.

Sejian, V. and Srivastava, R. S. 2009. Effects of melatonin on adrenal cortical functions of Indian Goats under thermal stress. Veterinary Medicine International, Pp. 1- 
6.

Sejian, V., Maurya, V. P. and Naqvi, S. M. K. 2010. Adaptive capability as indicated by endocrine and biochemical responses of Malpura ewes subjected to combined stresses (thermal and nutritional) in a semiarid tropical environment. International Journal of Biometereology, 54: 653-661.

Shrikhande, G. B., Rode, A. M., Pradhan, M. S. and Satpute, A. K. 2008. Seasonal effect on the composition of blood in cattle. Veterinary World, 1(11): 341-342.

Silanikove, N. 1992. Effects of water scarcity and hot environments on appetite and digestion in ruminants: a review. Livestock Production Science, 30: 175-194.

Silanikove, N. 2000. Effects of heat stress on the welfare of extensively managed domestic animals. Livestock Production Science, 67: 1-18.

Snedecor, G. W. and Cochran, W. G. 2004. Statistical Methods, 8 Edn., Oxford and IBH Pub. Co., Kolkata.

Soveri, T., Sankari, S. and Nieminen, M. 1992. Blood chemistry of reindeer calves (Rangifer tarandus) during the winter season. Comparative Biochemistry and Physiology, 102A(1): 191-196.

Sreedhar, S., Rao, K. S., Suresh, J., Moorthy, P. R. S. and Reddy, V. P. 2013. Changes in haematocrit and some serum biochemical profile of Sahiwal and Jersey $\times$ Sahiwal cows in tropical environments. Veterinarski Arhiv, 83(2): 171-187.

Srikandakumar, A., Johnson, E. H. and Mahgoub, O. 2003. Effect of heat stress on respiratory rate, rectal temperature and blood chemistry in Omani and Australian merino sheep. Small Ruminant Research, 49: 193-198.

Suhair, M. S. and Abdalla, A. M. 2013. Effect of season changes and shearing on thermoregulation, blood constituents and semen characteristics of desert ram (Ovis aries). Pakistan Journal of Biological Science, 16(24): 1884-1893.

Suhair, M. S. 2012. Effects of level of feeding and season on rectal temperature and blood metabolites in desert rams. Journal of Academy of Nutrition and Dietetics, 1(2): 14-18.

Thando, M. C. 2016. Influence of water stress on feed intake, growth performance and nutritional status of Nguni goats. PhD, thesis.

Tiffany, T. O., Jensen, J., Burtis, C. A., Overton, J. B. and Scott, C. D. 1972. Clinical Chemistry, 18: 829.

Toora, B. D. and Rajagopal, G. 2002. Measurement of creatinine by Jaffe's reaction--determination of concentration of sodium hydroxide required for maximum color development in standard, urine and protein free filtrate of serum. Animal, 40(3): 352-354.

Trinder, P. 1969. Determination of glucose in blood using glucose oxidase with an alternative oxygen acceptor. Annals of Clinical Biochemistry, 6: 24.

Zetingrao, G. N. 2003. Effects of water deprivation in Osmanabadi goats of Marathwada region. $\mathrm{PhD}$. Thesis.

\section{How to cite this article:}

Kour, S., J. Devi, K. Sarma and Chakraborty, D. 2020. Effect of Vitamin C on Some Biochemical Profile in Water Deprived Goats. Int.J.Curr.Microbiol.App.Sci. 9(10): 524-537. doi: https://doi.org/10.20546/ijcmas.2020.910.063 